\title{
Estudio de la vinculación que tienen los niños y niñas escolares con sus perros y los efectos socioemocionales de este vínculo
}

\section{The bond between school-age children and their dogs, and the socioemotional effects of this bond}

\author{
Claudia Schencke ${ }^{1} \quad$ Chamarrita Farkas \\ Pontificia Universidad Católica de Chile
}

(Rec: diciembre de 2011 - Acep: mayo de 2012)

\begin{abstract}
Resumen
Estudios internacionales avalan los beneficios de la tenencia de mascotas en la calidad de vida de los niños, sin embargo, en Chile no hay investigaciones sobre este tema. Por eso, el objetivo de este estudio fue investigar la vinculación que tienen los niños y niñas escolares con sus perros y los efectos sociemocionales de este vínculo, específicamente en relación al autoconcepto y percepción de apoyo social. Aparecen diferencias con resultados de investigaciones internacionales, ya que no se observa una diferencia significativa en autoconcepto o percepción de apoyo social entre niños con y sin perro. No obstante, aparece como significativo el que niños con un autoconcepto más bajo en popularidad muestran un vínculo más cercano con sus mascotas que aquellos que se perciben como populares.
\end{abstract}

Palabras clave: vínculo con mascotas, escolares, autoconcepto y apoyo social.

\begin{abstract}
Numerous international studies have proved that owning a pet can enhance the quality of life of school-age children; however, there have been no such studies in Chile. The objective of the present study is to analyzes the bond between school-age children and their dogs, and the socioemotional effects of such bond, in particular as it relates to self-identity and the perception of social support. Some differences appear in the results of international studies, in the sense that no significant difference can be observed in terms of self-identity or perceived social support between children who own a dog and children who don't. However, it is significant that children who perceive themselves as unpopular have a stronger bond with their pets than those who perceive themselves as popular.
\end{abstract}

Keywords: pets bonding, schoolers, self concept and social support.

\footnotetext{
${ }^{1}$ Correspondencia dirigida a: Claudia Schencke. Escuela de Psicología, Pontificia Universidad Católica de Chile, Avda. Vicuña Mackenna 4860, Macul, Santiago, Chile. E-mail: claudiaschencke@gmail.com.
} 


\section{Introducción}

Actualmente, la tenencia de mascotas es un fenómento masivo a nivel mundial. Estudios indican que el número de viviendas con mascotas es cercano al $62,9 \%$ en la provincia de Santiago de Chile, con un promedio de mascotas por vivienda de 1.2, siendo los más comunes los perros y luego los gatos (académicos de la UNICIT presentaron estudio sobre la población de animales domésticos en Santiago, 2003).

La tenencia de perros como mascotas es reconocida como beneficiosa para el desarrollo de los niños. Por ejemplo, se ha visto en estudios internacionales que los niños tienden a acudir a sus mascotas en los momentos de tensión emocional (Mc Nicholas \& Collins, 2001), o que los niños que tienen un vínculo con mascotas tienen puntajes más altos en empatía, autoestima y auto-concepto que aquellos que no tienen relación con mascotas (Poresky \& Hendryx, 1990; Van Houtte \& Jarvis, 1995, ambos citados en Barker, 1999). Además, se ha estudiado que los niños que ayudan a criar animales logran una mejor comprensión del lenguaje corporal y los motivos y sentimientos de los demás (Becker, 2003).

Sin embargo, en Chile no hay estudios que aborden este tema, considerando qué beneficios psicológicos tiene y en qué circunstancias se dan. Se han llevado a cabo estudios de mercado con fines comerciales y algunos enfocados en la problemática de los perros vagos como un tema de relevancia sanitaria, pero poco se ha estudiado en torno a la función que tiene la mascota para los miembros de la familia que la adopta, en especial para familias de niveles socioeconómicos más bajos.

La evidencia actual en torno al tema orienta a pensar que uno de los aspectos relevantes de la tenencia de mascotas es el apoyo social que otorga (Mc Nicholas \& Collins, 2001), sobre todo en el aspecto emocional, lo que representaría un factor protector, en especial para los niños que se encuentran en una situación de vulnerabilidad, característica de la pobreza. Empero, la literatura hace escasa referencia a las mascotas como posibles fuentes de apoyo social.

\section{Implicancias del vínculo con animales en la calidad de vida}

Diferentes estudios se han centrado en medir los efectos que tienen para el ser humano convivir con mascotas. En 1980, Friedman, Katcher y Lynch (citados en Friedmann, 2000) realizaron un estudio cuantitativo en que hacían seguimiento por período de un año a pacientes que habían sufrido infartos u otras afecciones cardiacas, distinguiendo entre aquellos que tenían mascotas y aquellos que no. Y observaron una diferencia estadísticamente significativa pues la tasa de mortalidad en el caso de los primeros era sustancialmente menor comparada con los segundos.

En Australia, con una muestra compuesta por 5.000 personas, Anderson, Reid y Jenings (1992, citados en Friedman, 2000) obtuvieron los mismos resultados, controlando variables tales como masa corporal, alimentación, hábitos de ejercicio y otros aspectos médicos relevantes. Serpell (2000) mostró que las personas con mascotas no aumentaban la cantidad de visitas al médico en período de crisis, a diferencia de aquellas sin mascotas, lo cual fue vinculado al apoyo social que daban los animales mediante su compañía.

Fogel (1986, citado en Noonan, 1998) estableció que los dueños de mascotas tenían necesidades afiliativas más fuertes y eran más responsivos que los que no tenían mascotas. Las mascotas permiten experiencias íntimas y de riqueza sensorial, como acariciar, abrazar, hablar, y hacen que sea socialmente aceptable jugar, regalonear, hacer el ridículo y acercarse a completos desconocidos para conversar.

Desde la perspectiva de los estudios en apoyo social, las mascotas son vistas como un tipo no-humano de apoyo, capaz de conferir salud y beneficios comportamentales a sus compañeros humanos (Garrity \& Stallones, 1998). Lo que las personas suelen valorar más en su relación con las mascotas, es la compañía que éstas les ofrecen (Hart, 2000). Garrity y Stallones (1998) concluyeron, tras realizar una revisión bibliográfica de veinticinco estudios empíricos en torno al tema, que los beneficios del contacto con mascotas son consistentes con los beneficios constatados en estudios de apoyo social humanos. Las mascotas ofrecen un sistema de apoyo incondicional, además de ser de relativamente bajo costo mantenerlas y sus demandas son simples y de bajo nivel de conflicto (Hart, 2000).

\section{La relación entre niños y mascotas}

Estudios internacionales han demostrado que los niños tienden a acudir a sus mascotas en los momentos de tensión emocional. En una investigación realizada por Mc Nicholas y Collins (2001) acerca de la percepción que tienen los niños sobre el apoyo social otorgado por sus relaciones personales, aparece que las mascotas puntúan más alto que ciertos tipos de relaciones humanas, caracterizándose, principalmente, por ser considerados proveedores de calma frente a situaciones de miedo o enfermedad, estima y ser buenos confidentes 
de un secreto. El estudio concluye que los niños son capaces de diferenciar entre las habilidades reales e irreales de los animales, y consideran a sus mascotas como miembros familiares cercanos no sólo por vivir en la misma casa, sino también por las funciones que cumplen.

Los niños que tienen un vínculo con mascotas tienen puntajes más altos en empatía, autoestima y auto-concepto, que aquellos que no tienen relación con mascotas (Poresky \& Hendryx, 1990; van Houtte \& Jarvis, 1995, ambos citados en Barker, 1999). La oportunidad que dan las mascotas de criar y cuidar de otro es vista como una forma de apoyo por Weiss (1974, citado en Collins \& Mc Nicholas, 1998), por incrementar la sensación de competencia y de estima, y de ser necesitado por otro. Becker (2003) asegura que "los niños que ayudan a criar animales entienden mejor el lenguaje corporal y los motivos y sentimientos de los demás" (p.31).

Se ha investigado que el contacto con animales estimula el habla y la interacción social en niños introvertidos y hasta mutistas (Becker, 2003; Sams, et al., 1999). Las personas son más estimuladas a iniciar una conversación, reír y compartir historias cuando están en presencia de un perro que cuando no lo están. Por otro lado, los animales en sí pueden ser compañeros conversacionales. Aunque no respondan de manera verbal, transmiten de forma suficientemente convincente su amor y afecto hacia sus compañeros humanos. Esto podría relacionarse con la idea de que hablar o tocar a una mascota es menos estresante que hablar o leerle a un humano (Hart, 2000).

Las mascotas también pueden tener un efecto importante como amortiguadores frente a situaciones de estrés. Demello (1999) realizó un estudio en el cual descubrió que la mera presencia de una mascota amistosa desconocida, puede aportar en la reducción de presión sanguínea y frecuencia cardiaca, tras el término de un estresor cognitivo, independientemente del reporte subjetivo, el género y la actitud hacia las mascotas (Demello, 1999).

Nagengast, Baun, Megel y Leibowits (1997, citados en Schaefer, 2002) estudiaron el nivel de umbral fisiológico y estrés conductual durante un examen físico de 23 niños entre 3 y 6 años. En comparación con grupos control, se descubrió que la presencia de un perro durante el examen contribuyó a bajar la media de presión arterial, presión sanguínea sistólica y distólica, y frecuencia cardíaca, además de aumentar la sensación de seguridad y disminuir las conductas de búsqueda de información, búsqueda de apoyo emocional y verbalizaciones de dolor durante el examen.
Friedmann, Katcher, Thomas, Lynch y Messen (1983, citados en Schaefer, 2002) descubrieron que la presencia de un animal podía contribuir a disminuir la ansiedad y la excitación fisiológica cuando los niños (entre 9 y 16 años) estaban en momentos de descanso y de actividad (leer en voz alta). Los resultados mostraron que la presencia del perro aportó significativamente a la disminución de los niveles de presión sanguínea y frecuencia cardíaca, tanto en descanso como durante la lectura.

\section{Comprensión psicológica de la relevancia de las mascotas en la infancia}

Barker (1999) plantea que las mascotas pueden, por medio de su presencia segura, fortalecer el sentimiento de confianza y seguridad, facilitando un sentimiento de autonomía y despertando interés en emprender cosas, por su rol como compañeros de juego activo. Por otro lado, potencian el descubrimiento del medio y favorecen el desarrollo de la paciencia y el autocontrol. Además, incrementan de manera positiva el espíritu de esfuerzo y eficacia, por ser entrenables y reaccionar a las instrucciones del niño, y favorecen el desarrollo de la identidad al ser camaradas y actuar como posibles figuras de apoyo emocional y social.

Según Levinson (1997), es por medio de la identificación con el animal que la mascota adquiere un valor tan significativo para el niño, y le permite incorporar las fortalezas de ésta. Para Caplan (1951, citado en Levinson, 1997), una probable razón de que los niños toman tan fácil y gustosamente a las mascotas como compañeros cercanos, tiene que ver con la necesidad del niño de cercanía a un sustituto de los padres que él puede controlar y dirigir como quiere. Freud sostenía que el vínculo de las mascotas, en especial perros, hacia los humanos carecía de ambivalencia, lo que representaba, a su parecer, un símbolo de amor y lealtad incondicional (Prothmann, 2006).

Por otro lado, el que los niños busquen a sus mascotas en momento de estrés o miedo, puede relacionarse con el concepto de objeto transicional de Winnicott (1953). El objeto transicional es en general algo suave, un pedazo de tela o un juguete. En el desarrollo normal del niño, éste va desarrollando otros intereses y su necesidad por el objeto transicional decrece hasta desaparecer, sin embargo, en tiempos de crisis o tensión emocional, el objeto transicional se convierte en una defensa contra la ansiedad (Stevenson, 1954, citado en Levinson, 1997). 
De acuerdo a los antecedentes revisados, esta investigación tuvo como objetivo estudiar la vinculación que tienen los niños y niñas escolares con sus mascotas perros y los efectos sociemocionales de este vínculo. Concretamente, su buscaba analizar el vínculo y la percepción de apoyo social que los niños reportaban tener con sus perros, y si había relación entre estos dos aspectos. Además, se quería estudiar si existían diferencias en autoconcepto y percepción de apoyo social, según si el niño tenía perro o no y según la calidad del vínculo con éste.

El rango etáreo de la muestra se determinó debido a que en la etapa escolar los niños y niñas están en condiciones de relacionarse de manera más cercana con sus mascotas. Dado que en esta etapa los niños ya tienen avances sociales, cognitivos y emocionales, es una excelente etapa para que ellos se relacionen con sus perros (Lookabaugh, 1998).

Estudiar la vinculación que tienen los niños y niñas escolares con sus perros y los efectos sociemocionales de este vínculo en la población chilena, resulta un aporte para la psicología, ya que pese a lo masivo que es el fenómeno de tener una mascota en la infancia en nuestro país, es bastante poco lo que se ha estudiado de manera sistemática respecto a la influencia de esta relación en el desarrollo de los niños. Esto adquiere mayor relevancia al ser realizado en un grupo de riesgo social, ya que un autoconcepto positivo y la percepción de apoyo social son reconocidos factores protectores en salud mental, por lo que estudiar el aporte de las mascotas en estas áreas podría aportar más información y abrir nuevos caminos de investigación en el área de promoción de salud mental, en un campo bastante nuevo, que aún se encuentra en una fase incipiente.

\section{Método}

\section{Diseño}

El presente estudio utilizó una metodología cuantitativa, caracterizándose por un diseño descriptivo, transversal, correlacional y comparativo.

\section{Participantes}

La muestra estuvo compuesta por 63 niños y niñas. Éstos fueron captados en escuelas municipales de la comuna de Peñalolén, región metropolitana. Los criterios de inclusión fueron que tuvieran un perro como mascota hacía al menos 6 meses (para el grupo estudio), y que no tuvieran otros animales como mascotas (tanto para el grupo de estudio como para el grupo control).

La media de edad de los niños era de 10,97 años (D.S $=0,761$ ), en un rango de 10 a 12 años. En la Tabla 1 se observa su distribución por edad y sexo.

Tabla 1

Descripción de los participantes por edad y sexo.

\begin{tabular}{lccc}
\hline Edad/Sexo & Masculino & Femenino & Total \\
\hline 10 años & 8 & 11 & 19 \\
11 años & 12 & 15 & 27 \\
12 años & 6 & 11 & 17 \\
Total & 26 & 37 & 63 \\
\hline
\end{tabular}

Los niños y niñas participantes del estudio asistían a 5 diferentes colegios municipales de la comuna de Peñalolén, cursando entre $4^{\circ}$ y $6^{\circ}$ básico. De los niños participantes, 32 de ellos fueron asignados al grupo estudio (GE) y 31 al grupo control (GC).

Respecto a las características de las familias de los niños y niñas participantes, la media de integrantes de la familia era de 5,9 (D.S. $=2,5$ ) y la media de hermanos era de 2 (D.S. $=1,3$ ). En un $70 \%$ de los casos, el padre vivía en el hogar.

En las familias del GE, la cantidad de perros en la casa alcanzó una media de 1,5 (D.S. = ,9) y el promedio de tiempo en que la mascota vivía en el hogar era de 52 meses (D.S. $=42,5$ ). La mayoría de los niños había tenido perro antes (66\%), al igual que sus padres $(72 \%)$. En un 56\% de los casos, el niño aparecía como el dueño del perro, y los cuidados del perro eran realizados en su mayoría por los adultos de la familia, a veces acompañados por los niños.

\section{Instrumentos}

Cuestionario sociodemográfico. Desarrollado especialmente para este estudio, orientado a recoger información para caracterizar la muestra.

Escala de autoconcepto de Piers-Harris (Piers \& Harris, 1984, adaptada en Chile por Gorostegui, M.E., 1992). Esta es una escala de autorreporte, destinada a evaluar el autoconcepto en niños y niñas desde 8 a 12 años, o escolares entre $3^{\circ}$ y $6^{\circ}$ año básico inclusive. Consta de 70 ítemes de preguntas cerradas, administrable tanto en forma individual como grupal. Arroja un puntaje total (dimensión total del autoconcepto, entre más alto el puntaje más positivo el autoconcepto) y seis subescalas (dimensiones específicas: conducta, estatus intelectual y escolar, apariencia y atributos 
físicos, ausencia de ansiedad, popularidad y felicidad/ satisfacción).

Test "Mi familia y mis amigos" ("My family and friends", Reid \& Landesman, 1989). Este instrumento evalúa la percepción de apoyo social en niños entre seis y doce años. Consiste en doce diálogos basados en prinicipios vigotskianos y entrega información acerca de las percepciones de disponibilidad de individuos en sus redes que les proveen diferentes tipos de apoyo social, y la satisfacción que tienen de la ayuda recibida (Reid \& Landesman, 1989). Si bien en este instrumento no se incluyen a las mascotas como posibles fuentes de apoyo social percibido, se incluyó en la consigna a las mascotas, lo cual ha sido realizado antes en un estudio de McNicolas y Collins (2001), con un instrumento equivalente a "My family and friends", pero para niños entre 7 y 8 años. Se optó por excluir de los análisis la situación 5, debido a que su redacción resultaba muy confusa para la comprension de los niños.

Escala de vínculo con la mascota (Companion animal bonding scale, CABS, Poresky, Hendrix, Mosier \& Samuelson, 1987). Este instrumento está compuesto por 8 ítems que miden el alcance de las actividades entre niño y animal. Se enfoca en la calidad de la relación entre la persona y la mascota, basado en evidencia que muestra el vínculo humano-animal. La confiabilidad interna de este instrumento es de 0.82 y 0,77 , respectivamente. La escala de vínculo con la mascota tiene puntajes de 1 a 5 , en la que a mayor puntaje representa mejor vínculo. Se estimó que un puntaje igual o sobre 3 podría ser considerado como un vínculo adecuado, debido a que este puntaje está por sobre el promedio del puntaje posible $(2,5)$.

\section{Procedimiento}

Inicialmente se envió la versión traducida de las escalas "Mi familia y mis amigos" y la escala de vínculo a tres jueces expertos para que revisaran la traducción y realizaran sugerencias. Paralelamente, se construyó el cuestionario sociodemográfico considerando posibles variables interesantes para el futuro análisis.

Una vez conformado el set de instrumentos a aplicar, se realizó la constatación de grupos cursos en las distintas escuelas de la comuna de Peñalolén. Los apoderados de los niños y niñas firmaron una carta de consentimiento informado y a los niños se les pidió asentimiento verbal.

La aplicación de los instrumentos se realizó en grupos de entre 3 a 5 niños, en una sala facilitada por el establecimiento. Todos los niños y niñas respondieron el cuestionario sociodemográfico, el test de Piers y Harris y el test "Mi familia y mis amigos". Posteriormente, sólo los niños que tenían perro respondieron la escala de vínculo con la mascota.

\section{Análisis de resultados}

Para el análisis de los datos se utilizó análisis de frecuencia para describir resultados, Correlación $\mathrm{r}$ de Spearman para la correlación de variables como apoyo social y calidad del vínculo con el perro, o autoconcepto y calidad del vínculo con el perro. Además se realizaron análisis comparativos por medio de ANOVA, para comparar GE y GC en percepción de apoyo social y autoconcepto. Por último, se ocuparon pruebas no paramétricas (U de Mann - Whitney) para comparar grupos con escaso número de participantes.

Tabla 2

Descripción de resultados de la escala de vínculo con la mascota.

\begin{tabular}{lccccc}
\hline Ítem & $\mathrm{N}$ & Mínimo & Máximo & Media & Desv. típ. \\
\hline Hacerse cargo de cuidar la mascota & 32 & 1 & 5 & 3,91 & 1,174 \\
Lavar o bañar a la mascota & 32 & 1 & 5 & 2,84 & 1,322 \\
Hacer cariño o regalonear & 32 & 2 & 5 & 4,44 & 1,014 \\
La mascota duerme en la pieza & 32 & 1 & 5 & 2,28 & 1,611 \\
Sentirse entendido por lamascota & 32 & 1 & 5 & 3,91 & 1,201 \\
Sentir una relación cercana con la mascota & 32 & 1 & 5 & 4,13 & 1,157 \\
Viajar con la mascota & 32 & 1 & 5 & 2,28 & 1,276 \\
Dormir en la misma cama con la mascota & 32 & 1 & 5 & 2,06 & 1,435 \\
Promedio & 32 & 1,38 & 4,63 & 3,2227 &, 84346 \\
\hline
\end{tabular}




\section{Resultados}

\section{Análisis descriptivo del vínculo con la mascota}

Como es posible observar en la Tabla 2, el promedio de los 8 ítems de la escala de vínculo con la mascota fue de 3,2 ptos. (D.S. = ,84), lo que es considerado un vínculo adecuado.

$\mathrm{Al}$ revisar cada caso particular, es posible apreciar que existen 4 situaciones en las cuales el vínculo con la mascota es adecuado: la frecuencia con que se le hace cariño o se regalonea la mascota, el sentir que se tiene una relación cercana con ella, el hacerse cargo de su cuidado y el sentir que su mascota los entiende (rango de 3,91 a 4,44) .

Los ítems que aparecen con puntaje más bajo que el promedio (vínculo escaso) son en orden decreciente: frecuencia con que lavan o bañan a su mascota, frecuencia con que la mascota duerme en la pieza, frecuencia con que viajan con su mascota y, por último, frecuencia con la que la mascota duerme con ellos en la cama (rango de 2,06 a 2,84).

\section{Análisis descriptivo de la percepción de apoyo social que tienen los niños de sus perros}

Del total de niños del grupo de estudio $(\mathrm{N}=32), 8$ (25\%) perciben al perro como un apoyo social en las distintas situaciones evaluadas. Tal como lo muestra la Tabla 3 , las situaciones en que es mencionado el perro como apoyo social son en orden decreciente: cuando quieren estar con alguien que los hace sentir feliz (frecuencia: 4, Media=4), cuando se quieren relajar (frecuencia: 3, Media=4,7), cuando quieren compartir sus sentimientos (frecuencia: 2, Media=3,5), cuando quieren estar con alguien que hace o dice cosas que lo hacen sentirse bien consigo mismo (frecuencia: 2, Media $=4,5$ ) y finalmente cuando necesitan ayuda (frecuencia: 1, Media $=5$ ).

La calidad del apoyo social percibido por los niños de parte de sus mascotas perros es en promedio de 4,25, lo que es considerado una calidad alta, teniendo en cuenta que el puntaje máximo para la calidad es de 5 puntos.

\section{Análisis comparativo entre apoyo social y vinculo con el perro en el GE}

Para conocer la relación entre apoyo social y vínculo con el perro, se comparó niños que tienen perro (GE) en relación a si perciben apoyo social de sus perros o no, estableciendo dos grupos: niños que tienen un perro como mascota y lo perciben como apoyo social en alguna situación (de acuerdo a lo descrito en la Tabla 3) y niños que tienen un perro como mascota pero que no lo mencionan como apoyo social. Así, el primer grupo quedó conformado por 7 niños, y el segundo por 25. Debido al escaso número de sujetos por grupo, se compararon ambos grupos con una prueba no paramétrica (U de Mann-Whitney). Los resultados arrojaron que los niños que tienen perro y que lo perciben como apoyo social tienen significativamente un mayor vínculo con sus mascotas (Media $=3,77$ ) que aquellos niños que no lo perciben como figura de apoyo social (Media $=3,07$ ) (U de MannWhitney $=38,500 ; \mathrm{p}=, 023$ ).

Tabla 3

Situaciones en que el perro aparece mencionado como apoyo social

\begin{tabular}{|c|c|c|c|c|c|}
\hline Situación & Descripción & Frecuencia & $\begin{array}{l}\text { Porcentaje } \\
\text { (Grupo total) }\end{array}$ & Porcentaje (GE) & $\begin{array}{l}\text { Promedio } \\
\text { Calidad }\end{array}$ \\
\hline 9 & $\begin{array}{c}\text { Cuando quieres estar con alguien } \\
\text { que te hace sentir feliz }\end{array}$ & 4 & 6,35 & 12,5 & 4 \\
\hline 3 & Cuando te quieres relajar & 3 & 4,76 & 9,38 & 4.7 \\
\hline 1 & $\begin{array}{l}\text { Cuando quieres compartir tus } \\
\text { sentimientos }\end{array}$ & 2 & 3,17 & 6,25 & 3.5 \\
\hline 12 & $\begin{array}{l}\text { Cuando alguien hace o dice cosas } \\
\text { que te hacen sentir bien contigo }\end{array}$ & 2 & 3,17 & 6,25 & 4.5 \\
\hline 7 & Cuando necesitas ayuda & 1 & 1,59 & 3,13 & 5 \\
\hline Total & & 12 & 19,04 & 37,51 & 4,25 \\
\hline
\end{tabular}




\section{Análisis correlacional entre autoconcepto y calidad del vínculo en los niños del GE}

Se realizaron correlaciones entre la calidad del vínculo que el niño(a) reporta tener con su mascota perro en relación a las distintas subescalas (conducta, estatus intelectual y escolar, apariencia y atributos físicos, ausencia de ansiedad, popularidad y felicidad) y escala total de autoconcepto, observándose que no habría correlación entre la calidad del vínculo que el niño(a) reporta tener con su mascota perro en relación a su autoconcepto.

\section{Análisis comparativo en percepción de apoyo social y autoconcepto entre niños del GE y GC}

\section{A. Análisis comparativo en percepción de apoyo social entre GE y $G C$}

De acuerdo a los resultados obtenidos, no se aprecia una diferencia significativa en la calidad de apoyo social percibido en niños que tienen perros y en aquellos que no tienen. Cabe considerar que estos resultados se refieren al apoyo social percibido en su globalidad (no diferenciando según la persona de quien se recibe el apoyo social).

\section{B. Análisis comparativo en autoconcepto entre GE y $G C$}

De acuerdo a los análisis realizados en este aspecto, no existirían diferencias significativas en el autoconcepto tanto general como en sus áreas específicas entre niños que tienen perro y aquellos que no lo tienen. Sin embargo, en el subtipo "popularidad" aparece una tendencia a favor del GC que habría que considerar ( $\mathrm{F}$ $=2,867 ; \mathrm{p}=, 096$ ).

Luego se pensó que la calidad del vínculo que los niños tenían con sus perros podría generar una diferencia en su autoconcepto, por lo cual se procedió a diferenciar a los niños del GE en vínculo adecuado y vínculo escaso. Esta distribución se hizo tomando como punto de corte los 3 puntos, es decir, 3 o más puntos fue considerado vínculo adecuado y menos de 3 puntos vínculo escaso, y se hicieron los estudios para comparar autoconcepto entre estos dos grupos.

Los resultados muestran una diferencia significativa entre los niños con vínculo adecuado versus escaso con sus mascotas en el área de popularidad ( $\mathrm{F}$ $=9,282 ; \mathrm{p}=, 005)$, correspondiéndole a los niños con un vínculo adecuado un autoconcepto más bajo en el área de popularidad (M 0 49,29). Estos resultados son interesantes y se discuten posteriormente.

\section{Discusión}

Un primer objetivo de este estudio fue analizar el vínculo que los niños y niñas escolares reportaban tener con sus perros. Se observó que este vínculo en general es adecuado, sin embargo, esto no es válido para todas las escalas del cuestionario. Hay cuatro aspectos en los que el vínculo se muestra adecuado (la frecuencia con que se le hace cariño o se regalonea la mascota, el sentir que se tiene una relación cercana con ella, el hacerse cargo de su cuidado y el sentir que su mascota los entiende) y otros cuatro aspectos en los que el vínculo aparece como escaso (frecuencia con que lavan o bañan a su mascota, frecuencia con que la mascota duerme en la pieza, frecuencia con que viajan con su mascota, y por último, frecuencia con la que la mascota duerme con ellos en la cama).

$\mathrm{Al}$ observar estos resultados, es interesante poder analizar esta diferencia entre los aspectos que aparecen adecuados y aquellos en que aparece un vínculo escaso. Acerca de a los que aparecen como más altos, es posible observar un elemento afectivo en común, pues se destaca una relación de cariño, cercanía y de comprensión. Los que aparecen más bajos pueden estar relacionados con estilos familiares y características contextuales de trato hacia los perros, en las que los cuidados de éstos muchas veces incluyen sólo lo básico como alimentarlo, pero donde otros cuidados como bañarlo, pasearlo o que duerman dentro de la casa están menos considerados. Lo mismo para los viajes, ya que es posible comprender que de acuerdo a las características de las familias a las que pertenecen los niños de la muestra, nivel socioeconómico bajo y en general en la realidad chilena, llevar a una mascota a un viaje es algo poco común. También es posible considerar que en estas familias no se concibe que el perro pueda dormir en la pieza o en la cama del niño; el perro duerme afuera, pero esto no significa falta de cariño. Es posible relacionar estos resultados con aspectos socioculturales de la relación con las mascotas, por lo que es importante tener cautela con los instrumentos de evaluación desarrollados en otros países, en los que esta relación es diferente.

Estos aspectos se observaron de manera cualitativa cuando los niños respondían la parte del cuestionario sociodemográfico en la que se les preguntaba por el miembro de la familia encargado de alguno de los 
cuidados básicos de los perros. Algunos de ellos manifestaron que sus mascotas no recibían esos cuidados, relatando por ejemplo que las mascotas pasaban el día en la calle y que sólo llegaban a la casa a comer, no eran bañadas o bien que no eran llevadas al veterinario. En este sentido, aparece entre los niños del estudio una relación de cuidados básicos, posiblemente relacionado con que el perro como mascota de una familia de escasos recursos puede adquirir una prioridad más baja que otros factores, invirtiéndose menos recursos económicos en ella. Empero, esto no afectaría la percepción de cercanía que los niños sienten de parte de sus perros.

Al analizar la percepción de apoyo social que los niños tienen respecto de sus perros, los resultados muestran que el $25 \%$ de los niños percibe recibir apoyo social de parte de sus mascotas perro. Cuando perciben apoyo social de parte de sus perros, este respaldo es adecuado y se daría en situaciones puntuales: cuando quieren estar con alguien que los hace sentir feliz, cuando se quieren relajar, cuando quieren compartir sus sentimientos y cuando quieren estar con alguien que hace o dice cosas que los hace sentirse bien consigo mismos.

Al observar con más detención esta área, resalta como aspecto común nuevamente el contenido afectivo de la relación con la mascota. Estos resultados son consistentes con la bibliografía del tema, la cual hace hincapié en la importancia que tiene la relación con las mascotas por la incondicionalidad del vínculo y el aspecto lúdico y recreativo de la relación entre niños y mascotas en la edad escolar. Uno de los aspectos más valorados por las personas respecto a la relación con sus mascotas es la compañía y el afecto que éstas los ofrecen (Hart, 2000), lo que implica un aporte importante considerando que los beneficios del contacto con mascotas son consistentes con los beneficios constatados en estudios de apoyo social humanos (Garrity y Stallones, 1998). Esto también se condice con los estudios que se han realizado en Chile respecto a la preferencia por los perros como mascota, lo que tiene relación con motivos emocionales, puesto que éstos serían considerados más idóneos para otorgar compañía, cariño y seguridad (Collect GFK, 2007).

Resultaría interesante continuar con esta línea de investigación, considerando lo masiva que es la tenencia de mascotas en Chile, y lo relevante que es para la salud mental el contar con figuras de apoyo social, especialmente en sectores más vulnerables. Esto adquiere mayor relevancia si consideramos que las mascotas podrían representar un recurso adicional como sistema de apoyo al que se puede recurrir a cualquier hora del día o de la noche, cuando familiares o amigos no están disponibles, además de ser de relativamente bajo costo mantenerlos y sus demandas son simples y de bajo nivel de conflicto (Hart, 2000).

En cuanto a la relación entre apoyo social y vínculo con la mascota, los resultados arrojan que no habría una relación entre el vínculo con la mascota y la percepción de apoyo social que los niños tienen en forma global. Pese a esto, aparece que los niños que tienen perro y que lo perciben como apoyo social, tienen un mayor vínculo con ellos. En este sentido, es posible pensar que los niños que perciben por parte de sus mascotas perros apoyo social, tienden a establecer relaciones más cercanas con sus mascotas. En este punto se abren nuevos cuestionamientos acerca de qué estaría influyendo en que para algunos niños los perros sean percibidos como apoyo social y para otros no, considerando que la percepción de apoyo social por parte del perro es relevante para establecer un vínculo con éste. Aquí queda claro que la percepción que el niño tiene de su perro es más relevante y no basta sólo el hecho de tener perro o no. Una posible explicación para esto tendría relación con lo que señala Fogel (1986, citado en Noonan, 1998), quien establece que los dueños de mascotas que se vinculan con ellas tienen necesidades afiliativas más fuertes y son más responsivos, es decir, podrían haber características de personalidad a la base de esta vinculación. Otra posible explicación estaría dada con las condiciones vitales en las que se encuentran los niños. En estudios internacionales acerca de la percepción de apoyo social por parte de las mascotas aparece que éstas puntúan más alto que ciertos tipos de relaciones humanas en apoyo social, caracterizándose, principalmente, por ser considerados proveedores de calma frente a situaciones de miedo o enfermedad, estima y ser buenos confidentes de un secreto (Mc Nicholas \& Collins, 2001). ¿Es posible pensar que esta percepción es situacional? ¿Puede ir este vínculo cambiando con el tiempo y de acuerdo a los eventos vitales? Estas preguntas deben ser consideradas en futuros estudios.

En cuanto a las diferencias en autoconcepto según la calidad del vínculo con la mascota, los resultados de este estudio no establecen tal relación. Respecto a este punto no hay literatura que describa investigaciones previas al tema, ni a nivel internacional ni nacional, sólo es nombrado informalmente en algunos textos. En este sentido, es importante aclarar que esta es una hipótesis exploratoria, pero que los resultados de este estudio no permiten avalar esta idea. 
Sobre la diferencia entre los niños que tienen perro y aquellos que no en cuanto al autoconcepto, los resultados de este estudio no muestran que existiría tal relación, lo cual es discordante con algunas investigaciones de otros países, que establecen que los niños que tienen un vínculo con mascotas tienen puntajes más altos en empatía, autoestima y autoconcepto, que aquellos que no tienen relación con mascotas (Poresky \& Hendryx, 1990; van Houtte \& Jarvis, 1995, ambos citados en Barker, 1999).

Una posible explicación para estos resultados se debe a las características de la muestra. Los niños y niñas que participaron en el estudio pertenecen a colegios municipales de la comuna de Peñalolén, los que tienen un alto índice de vulnerabilidad social relacionado a la situación de pobreza, por lo que el vínculo con una mascota podría resultar insuficiente para tener un efecto significativo en sus autoconceptos, considerando el contexto en el que se encuentran. En otras palabras, podrían haber otras variables en juego que estarían interfiriendo en la influencia positiva que tienen las mascotas en aspectos como el autoconcepto, características familiares, características de los ambientes escolares, etc.

De acuerdo a los análisis, no existirían diferencias significativas en el autoconcepto específico y general entre niños que tienen perro y aquellos que no lo tienen. Lo mismo aparece en relación al apoyo social percibido en forma global por los niños, el cual no cambia significativamente al tener o no un perro como mascota.

Sin embargo, en el área del autoconcepto, en el subtipo "popularidad" es posible observar que los niños que tienen un peor autoconcepto reportaron tener un vínculo adecuado con su mascota, mientras que los niños que se consideran más populares aparecen con un vínculo escaso con sus perros mascotas. Este resultado surge al separar a los niños que tienen un perro como mascota en dos grupos: aquellos que lo consideran como un apoyo social y aquellos que no lo consideran como tal.

Este resultado es posible comprenderlo desde la búsqueda de un refugio en sus mascotas por parte de estos niños que se sienten menos populares entre sus pares. Recordemos que uno de los aspectos centrales de la relación de la mascota es la incondicionalidad y la falta de juicio que hay en la relación con ellas. Los animales, en especial perros, no juzgan, no exigen, sino que aceptan incondicionalmente (Levinson, 1997).

Este estudio representa un aporte, ya que implica un primer abordaje de la temática de las mascotas desde la psicología a nivel nacional, abriendo un nuevo campo de investigación. Si bien existen estudios relacionados con el tema en otros países, muchos de ellos no cuentan con evidencia científica clara y eso contribuye a presentar una visión simplista y al desarrollo de mitos. Es posible concluir con este estudio que si bien la tenencia de mascotas tiene efectos en el desarrollo socioemocional de los niños en edad escolar, éstos no son generalizables y se dan en condiciones y aspectos puntuales, requiriéndose de futuros estudios que permitan revisar estos aspectos.

Es por ello que podría ser valioso profundizar mediante un estudio de tipo cualitativo algunos de los resultados más interesantes de esta investigación, como por ejemplo acerca del concepto que los niños tienen de sus mascotas, o poder realizar un estudio comparativo con niños pertenecientes a un nivel socioeconómico más alto.

Posibles limitaciones a este estudio tienen relación con el número reducido de la muestra, lo que implica un análisis acotado de los resultados. Por otro lado, para futuras investigaciones sería importante considerar la extensión de los instrumentos de evaluación, ya que se observó en algunos niños fatiga al responderlos o bien dificultades en la comprensión de las instrucciones. Por ejemplo en la escala "Mi familia y mis amigos" los niños mostraron algo de fatiga y aburrimiento al responder. Asímismo, es importante remarcar que este instrumento no incluye originalmente a las mascotas como posibles fuentes de apoyo social percibido, por lo que posiblemente los ítems dejen fuera otros aspectos en los que hubiese sido posible que los niños percibieran apoyo social de parte de sus perros, y que los ítems incluidos hubieran sido excesivamente centrados en humanos. Esto puede haber influido en los resultados.

Además, es primordial considerar variables como el tiempo en que tenían al perro, y si esto tenía una correlación con apoyo social, asimismo si habían tenido perro antes y de quién había sido el perro. Si bien algunos de estos datos estaban en el cuestionario sociodemográfico, no se analizaron debido al tamaño muestral, pero son variables moderadoras a tener en cuenta en futuros estudios.

Aunque si bien las hipótesis de este estudio fueron exploratorias y los resultados deben ser y Tomados con cautela, representa una primera aproximación nacional al tema de los aportes socioemocionales que conlleva la tenencia de mascota para los niños y abre este campo en el ámbito de la investigación en psicología. 


\section{Referencias}

Barker, S. (1999). Therapeutic aspects of the human-companion animal interaction. Psychiatric Times. 16 (2), p.89-96.

Becker, M. (2003). El poder sanador de las mascotas. Bogotá: Norma S.A.

Collect GFK (2007). Hábitos de tenencia de mascotas de los chilenos: Estudio de mercado. Santiago: Chile.

Collins, G. y McNicholas, J. (1998). A theoretical basis for health benefits of pet ownership: attachment versus psychological support. En Wilson y Turner (Eds.). Companion animals in human health (pp. 105-122), Orlando, Estados Unidos. California: Sage.

Demello, L. (1999). The effect on the presence of a companionanimal on physiological changes following the termination of cognitive stressors. Psychology and Health, 14, 859-868.

Académicos de la UNICIT presentaron estudio sobre la población de animales domésticos en Santiago (2003, 8 abril). Extraído el 20 de enero del 2008 desde: http://noticias.universia.cl/ vida-universitaria/noticia/2003/04/08/356163/academicosunicit-presentaron-estudio-poblacion-animales-domesticossantiago.html.

Friedmann, E. (2000). The Animal-Human Bond: Health and Wellness. En Audrey Fine (Eds.). Handbook on Animal Assisted Therapy, Theoretical Foundations and Guidelines for Practice (pp. 41-56), Orlando, Estados Unidos.

Garrity, T. y Stallones, L. (1998). Effects of Pet Contact on Human Well-Being. En Wilson \& Turner (Eds.). Companion animals in human health (pp. 135-148), Orlando, Estados Unidos. California: Sage.

Gorostegui, M. E. (1992). Adaptación y construcción de normas de la escala de autoconcepto para niños de Piers-Harris. Memoria para optar al Título de Psicólogo. Facultad de Ciencias Sociales. Pontificia Universidad Católica de Chile.

Hart, L (2000). Psychosocial Benefits of Animal Companionship. En Audrey Fine (Eds.). Handbook on Animal Assisted Therapy, Theoretical Foundations and Guidelines for Practice (pp. 59-76), Orlando, Estados Unidos.
Levinson, B. (1997). Pet-oriented child psychoterapy (2a ed.). Spriengfield, IL: Thomas.

Lookabaugh, S. (1998). The relationship between attachment to companion animals and self esteem: a development perspective. En Wilson \& Turner (Eds.). Companion animals in human health (pp. 135-148), Orlando, Estados Unidos. California: Sage.

McNicholas, J. y Collins, G.M. (2001). Children's representations of pets in their social networks. Child: Care, Health and Development. 27 (3), 279-294.

Noonan, D. (1998). People and pets. Psychodynamic Counselling, 4 (1), February, 17-31.

Piers, E. (1984). Piers-Harris Children's Self Concept Scale. Revised Manual. California: WPS.

Poresky, RH., Hendrix, C., Mosier, J. E. y Samuelson, M. L. (2006). Companion animal bonding scale, CABS. En Anderson, D (comp.). Assesing the Human-Animal Bond: a compendium of actual Measures, (p. 26-32).

Prothmann, A. (2006). Tiergestützte Kinderpsychoterapie. Peter Lang: Frankfurt.

Reid, M., Landesman, S., Treder, R. y Jaccard, J. (1989). "My family and friends": six to twelve-year-old children's perceptions of social support. Child Development, 60 (4), pp. 896-910.

Sams, M. J., Gardner, A. A., Sides, J. M., Smith, H. S., Street, M. W., Reed, C., Haynie, K. L. y Willenbring, S. (1999). Differential Behavioral Responses in autistic children: Animal Assisted Therapy versus conventional therapy. Extraído el 5 de septiembre del 2004 desde http://www.deltasociety.org.

Schaefer, K. (2002). Human-animal interactions as therapeutic intervention. Counseling and human development, 34 (5), 1-18.

Serpell, J. (2000). Animal Companions and Human Well-Being: An Historical exploration of the value of human-animal relationship. En Fine, A. (Ed.). Handbook on Animal-assisted therapy: theoretical foundations and guidelines for practice. (pp.115-129). San Diego, CA: Academic Press.

Winnicott, D. (1953). Transitional objects and transitional phenomena. International Journal of Pshychoanalysis, 24, 88-97. 